\title{
Evaluation of pathogenesis and biofilm formation ability of Yersinia pestis after 40-day exposure to simulated microgravity
}

Ye Li

Beijing Institute of Microbiology and Epidemiology

Yulu Chen

Beijing Institute of Microbiology and Epidemiology

Lei Wang

Beijing Institute of Microbiology and Epidemiology

Yixuan Li

Beijing Institute of Microbiology and Epidemiology

Ruifu Yang

Beijing Institute of Microbiology and Epidemiology

Yanping Han ( $\sim$ hypiota@hotmail.com )

Beijing Institute of Microbiology and Epidemiology

\section{Research Article}

Keywords: Yersinia pestis, simulated microgravity, virulence, cytotoxicity, biofilm

Posted Date: February 24th, 2021

DOI: https://doi.org/10.21203/rs.3.rs-226711/v1

License: (c) (1) This work is licensed under a Creative Commons Attribution 4.0 International License. Read Full License

Version of Record: A version of this preprint was published at International Journal of Astrobiology on February 28th, 2022. See the published version at https://doi.org/10.1017/S1473550422000039. 


\section{Abstract}

Background: With the increase of manned space missions and the rise of space microbiology, the research of microbes grown under microgravity environment attracts more attentions. The research scope in space microbiology has been extended beyond pathogens directly related to spaceflight $Y$. pestis, the causative agent of plague, is also of interest to researchers.

Results: After Y. pestis strain 201 cultivated for 40 consecutive passages in either simulated microgravity and normal gravity (NG) conditions, the cultures were used to observe the main phenotypic features of $Y$. pestis. By using crystal violet staining assays, increased biofilm amount was detected in $Y$. pestis grown under SMG condition. Besides that, the damage degrees of Hela cell caused by SMG-grown Y. pestis were found diminished in relative to those NG condition. Consistent with this observation, death course was delayed in mice infected with SMG-grown $Y$. pestis, suggesting that microgravity condition could contribute the attenuated virulence. RNA-seq-based transcriptomics analysis showed a total of 219 genes were differentially regulated, of which 92 upregulated and 127 downregulated. We found dozens of virulence-associated genes were downregulated, which partially explained the reduced virulence of Y. pestis under SMG condition. Our study demonstrated that long-term exposure to simulated microgravity influence the pathogenesis and biofilm formation ability of $Y$. pestis in a different way, which provides a novel avenue to study the mechanism of physiology and virulence in this pathogen.

Conclusions: Microgravity enhanced the ability of biofilm formation of $Y$. pestis. The virulence and cytotoxicity of $Y$. pestis were reduced under the microgravity environment. The expressions of many virulence-associated genes of $Y$. pestis were differentially regulated in response to the stimulated microgravity.

\section{Background}

Long-term exposure to the outer space environment may have blunted the immune functions of astronauts, which can increase the risk of infectious diseases caused by certain pathogenic microbes [1,2]. Microgravity is a key factor of the outer space environment, where microbes can adapt and respond to the special environment stress through gene modification and phenotypic alterations [3]. Due to expensive cost and limited space, high-aspect ratio rotating-wall vessels (HARVs) developed by NASA has been applied to cultivate microbes and thus provide a low-shear simulated microgravity (LSSMG) in the conventional laboratory [4-6]. Salmonella Typhimurium grown under microgravity exhibited increased virulence and resistance to acid stress and macrophage killing [7, 8], while diminished virulence was found in Staphylococcus aureus grown under microgravity condition [9]. These results suggest different microbes respond differently to the microgravity.

The genus Yersinia, a member of the family Enterobacteriaceae, is composed of 11 species, three of which ( $Y$. pestis, Y. pseudotuberculosis, and $Y$. enterocolitica) are pathogenic for rodents and humans. The most notorious species, $Y$. pestis, is the causative agent of plague. The deadly pathogen is always maintained in nature dependent upon cyclic transmission between fleas and mammals. $Y$. pestis is regarded as a model pathogen for studying the mechanism of vector-mediated dissemination and pathogenesis.

Surprisingly, two non-pathogenic members of Yersinia genus, $Y$. frederiksenii and $Y$. intermedia have been found during several spaceflights of International Space Station [10]. Low-shear force might be a common stress upon exposure of $Y$. pestis to spaceflight board or systematic infection within human body and flea proventriculus. Previously, the Rosenzweig's group has initiated studies on the effect of microgravity on $Y$. pestis growth physiology and virulence potential. Y. pestis exhibited impaired ability of cytotoxicity but no alterations in growth kinetics, cold-resistance, or antibiotic-sensitivity [11, 12]. Blood meals of fleas might provide the low-shear-force-like microenvironment to $Y$. pestis. The blocked bacterial masses in the flea proventriculus due to biofilm formation further contribute to the subsequent spread of mammalian infection. However, biofilm formation ability could not be observed in $Y$. pestis KIM derivative strain lacking of the pigmentation locus pgm [11]. How Y. pestis respond to mid-long-term microgravity stress remains to be investigated. Based on these considerations, here we used the $Y$. pestis microtus strain 201, which is avirulent to humans but highly lethal to mice, to evaluate the strain-specific effects of stimulated microgravity lasting for 40 days on virulence and biofilm production.

\section{Results}

\section{Increased biofilm formation ability of $Y$. pestis grown under SMG}

The biofilm formation ability was assessed by evaluating the amount of biofilm formed and adhered on the silicified gas-permeable membrane on the back of the bioreactors. We found that the amount of biofilm in SMG bioreactor was more than that in NG bioreactor (Figure 1A).

In order to quantitatively evaluate the biofilm formation ability, the $\mathrm{OD}_{570 \mathrm{~nm}}$ values of crystal violet staining divided by the $\mathrm{OD}_{620 \mathrm{~nm}}$ values of cultures in the 24-well plates before staining. This operation eliminated the influence of the different numbers of bacterial in each well. By calculating the ratio of $\mathrm{OD}_{570 \mathrm{~nm}}$ to $\mathrm{OD}_{620 \mathrm{~nm}}$ values, the SMG group had higher values than NG group ( 109 and 74.3 of mean value in the SMG and NG group, respectively)(Figure $1 \mathrm{~B}, p \otimes 0.05$, using Student $t$ test), indicating that SMG condition increased the biofilm formation ability of $Y$. pestis.

\section{No statistical difference in RAW 264.7 cells intracellular survival assay}

In RAW 264.7 cells intracellular survival assay, the trend of SMG group was almost consistent with NG group (Figure 2A). The mean values of survival rate at 8-hour past infection (hpi) were 2.85\% (SMG) and 2.47\% (NG), respectively. The survival rates of Y. pestis in RAW 264.7cells at 8-hpi had no statistically significant difference ( $p=0.5835$, using the Student $t$ test) between SMG and NG groups (Figure 2B). The figure of 8 -hpi was just the representative result in 
that the same trend was shown at other time points. This result indicated that the microgravity environment cannot change the viability of $Y$. pestis in macrophages.

\section{SMG reduced cytotoxicity of $Y$. pestis-induced HeLa cell}

The normal morphology of HeLa cells was irregular fusiform, while the infected HeLa cells were shown as varying degrees of rounding. At a MOI of $\sim 50$, approximately $23 \%$ of SMG-grown Y. pestis-infected HeLa cells (Figure 3A) were extremely rounded relative to $68 \%$ of NG-grown counterpart (Figure 3B), indicating that SMG reduced the cytotoxicity of $Y$. pestis to some extent.

The xCELLigence real-time cell analysis (RTCA) system could be used to quantitatively assess the degree of cytotoxicity[13]. Due to differences in cell growth in the first $12 \mathrm{~h} \otimes F i g u r e 4 A \rrbracket$, the cell index was normalized at the 12-hour point to eliminate the influence of different cultivated cell number of each well. The cell index data per 2 hours from 3-hour to 36-hour point was reanalyzed using GraphPad Prism 5.0 software (Figure 4B). Apoptosis of HeLa cells appeared under SMG condition was relatively less than NG condition, indicating that SMG weakened the cytotoxicity of $Y$. pestis.

The xCELLigence real-time cell analysis (RTCA) system could be used to quantitatively assess the degree of cytotoxicity[13]. Due to differences in cell growth in the first $12 \mathrm{~h} \otimes F i g u r e 4 A \rrbracket$, the cell index was normalized at the 12-hour point to eliminate the influence of different cultivated cell number of each well. The cell index data per 2 hours from 3-hour to 36-hour point was reanalyzed using GraphPad Prism 5.0 software (Figure 4B). Apoptosis of HeLa cells appeared under SMG condition was relatively less than NG condition, indicating that SMG weakened the cytotoxicity of $Y$. pestis.

\section{Discovery of the differential expressions of $\mathbf{2 1 9}$ genes between SMG and NG groups}

To identify the differential expressions of genes which were regulated by SMG condition relative to NG condition, RNA-seq and quantitative RT-PCR technologies were used to compare the transcripts between SMG and NG groups (The raw data of RNA-seq had submitted to the SRA database on NCBI, Accession to cite for these SRA data: PRJNA700490). According to the RNA-seq data, a total of 219 genes were found, including 92 upregulated and 127 downregulated genes which were indicated by at least a 2 -fold change in expression (with $95 \%$ confidence) in the SMG group relative to NG group. According to the KEGG pathway database, part of the differentially regulated genes were classified into 32 functional classes (Table 1), of them most were related to biosynthesis and material metabolism. We also found some genes relative with virulence expressed differently, such as YP_RS21365 (yopE), YP_RS21080 (yopH) in Yersinia infection pathway and YP_RS19170 (virG/ yscW) in bacterial secretion system pathway.

Table 1. KEGG pathway of differentially regulated genes in Y. pestis under SMG condition. 


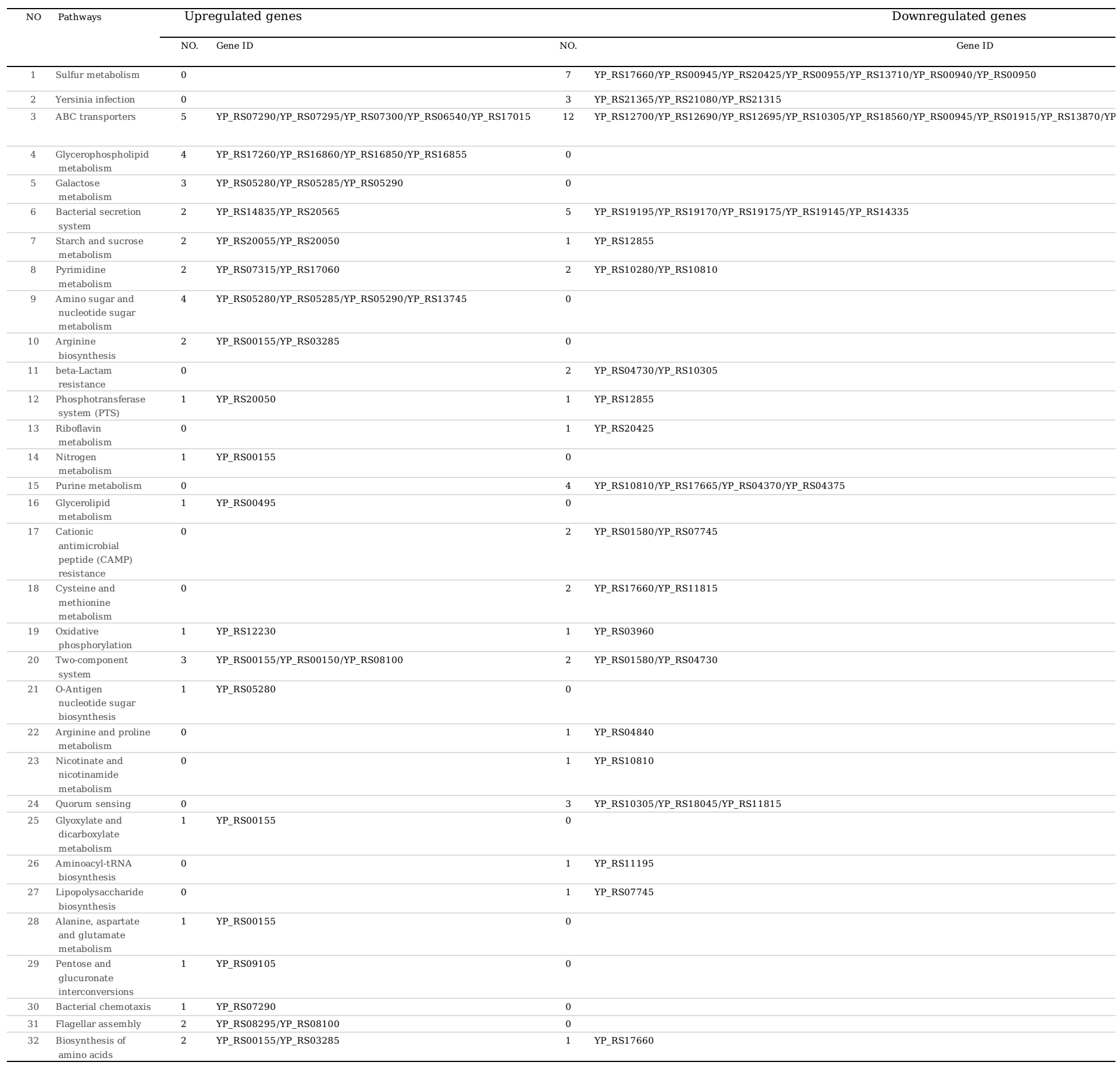

Of all the differentially expressed genes, we noticed that dozens of well-characterized virulence genes of $Y$. pestis were downregulated, including genes responsible for Type-III secretion system (yopH, yopE, yscW, yscA, yscB, IcrH), for iron metabolism (fyuA, irp and ybt cluster), caf1 operon encoding capsular antigen and the pla gene encoding plasminogen-activating protease. The downregulation of these genes might partially account for the impaired virulence observed in cell cytotoxicity and mice infection assays. Seven genes were selected and performed for quantitative RT-PCR (Table 2). The RT-PCR result correlated well with the RNA-seq data as shown in Figure 6, although the relative values were obtained higher based on RT-PCR than that on RNA-seq.

Table 2. Primers of the target genes used in qRT-PCR

\begin{tabular}{|c|c|}
\hline Gene name & Primers (forward / reverse, 5'-3') \\
\hline psaA & ACTGTCAAGCAGGGAAACAC/ ACCAACATAGTCACCATCGG \\
\hline udp & ACСТTCTACСТCСATTGCCG / TGAATAGCCСCTGTTGTCCC \\
\hline tsSC & ATATGGGCAGTTTGGTGGGG/ ATGCTTGGGGAAGAAGAGGT \\
\hline yopH & AATCTCTGTTCCTGTGGTTC/ TATTGCGTTTTGTTTCTGCT \\
\hline yscW & TGGGTTGTATGAGGGGAAAG/TAAGCTTTTGCTGAGCCACCGCCTG \\
\hline caf1 & CCTAAGGTAAACGGTGAGAA/ ATTGAGCGAACAAAGAAATC \\
\hline pla & CTTTATGACGCAGAAACAGG/ AAATGAGTATGGATCCCAGG \\
\hline 16 s rRNA & GAGCGGCGGACGGGTGAGTA/GGGCACATCTGATGGCATGA \\
\hline
\end{tabular}

\section{Discussion}


In recent years, microgravity-induced responses of many pathogenic bacteria have been widely characterized. The research scope in this field has been extended beyond pathogens directly related to spaceflight $[10,14]$. Phenotypic variation accelerated by stimulated microgravity or spaceflight broadens our insights into virulence mechanisms of various bacteria. In this study, SMG-induced changes of both pathogenesis and biofilm formation were observed in $Y$. pestis strain 201 grown for 40 successive passages within the HARV bioreactor. In agreement with the research finding of Rosenzweig's group focusing on short-term effect of SMG on $Y$. pestis cells [11, 12], the degree of HeLa cells damage were reduced to some extent upon infection of SMG-grown $Y$. pestis compared with NG-grown counterpart. The decreased cytotoxicity could be mainly attribute to repression of type III secretion system (T3SS) function, which allows $Y$. pestis to inject the effector proteins into the cytosol of eukaryotic cells $[15,16]$ and thus impede the phagocytosis and killing from host cells $[17,18]$. These speculations were also corroborated by our results of RNA-seq data.

In addition to the differential expression of the T3SS-related genes, we also observed the changes in the expression of many virulence factors of $Y$. pestis strain 201 such as pH6 antigen [19], Pla [20, 21], F1 antigen [22] and other iron-metabolism-related proteins (Ybt and FyuA) [23, 24] under SMG condition. The differential expression of these genes might be implicated in virulence attenuation of SMG-grown $Y$. pestis. However, this alteration of virulence phenotype was not observed in mice challenged with $1 \times 10^{8} \mathrm{CFU}$ of NG- and SMG-grown $Y$. pestis KIM/D27 by Rosenzweig's group. Similarly, the bacterial cultures of $20^{\text {th }}$ passage used to infect mice failed to attenuate the virulence (data not shown). In spite of different dose administration and different strain usage between two studies, we are more inclined to speculate that the long-duration maintenance under SMG might be more important contributor for the virulence alteration.

Biofilm is a membrane formed by the adhesion of polysaccharides, lipoproteins, fibrin and other substances secreted by bacteria and it can improve the ability of bacteria to adapt to the environment, anti-phagocytosis and drug resistance [25-27]. Enhanced ability of bacterial biofilm formation under SMG condition has been reported in Salmonella, K. pneumoniae and etc. [4, 28]. It is generally supposed that the ability of biofilm formation is positively correlated with bacterial pathogenesis. However, the opposite trend of biofilm formation ability and virulence was seen in SMG-grown $Y$. pestis. This phenomenon might be explained by the complex life cycle of $Y$. pestis, in which fleas are used as vectors to involve in bacterial dissemination. $Y$. pestis occurring in natural foci relies on the complex network of dissemination between wild rodents and fleas. Y. pestis colonizes the flea guts and gradually forms thick biofilms that block the flea proventriculus. The constant biting of the starving fleas injects the blood containing $Y$. pestis into new host bodies, which promotes the spread of plague [29]. We noticed however, that the putative biofilm-related genes of $Y$. pestis didn't exhibit the differential expressions.

\section{Conclusions}

In this study, we used HARV to study the phenotypic changes of $Y$. pestis in a microgravity environment. SMG condition reduces the expression of plaguerelated virulence genes, resulting in weakened virulence, which is different from other Gram-negative bacteria. The blood cells can maintain a continuous suspension state, indicating that bacteria could also face with a microgravity-like environment during the process of disseminating with blood. Therefore, research on pathogens in the microgravity environment is helpful to study their changes in the body [12]. Since the regulation of $Y$. pestis biofilm is a very complicated process, and post-transcriptional regulation is considered to be the most important process [30], our future work will focus on relevant sRNAs to explore the potential effects on enhanced biofilm formation ability of $Y$. pestis under SMG condition.

\section{Methods}

\section{Bacterial strains and culture conditions}

Yersinia pestis microtus strain 201 is isolated from Microtus brandti and is extremely lethal to mice but avirulent to humans and other large animals[31]. $Y$. pestis wild-type (WT) strain 201 is reserved in our lab, and used in this study. In general, Y. pestis is grown in lysogeny broth (LB) medium or agar plate at $26^{\circ} \mathrm{C}$ or $37^{\circ} \mathrm{C}$, unless otherwise specified. Resuscitated $Y$. pestis microtus strain 201 was inoculated at a dilution of 1:20 into the HARV bioreactors. When HARVs were filled with $\sim 58 \mathrm{ml}$ fresh LB medium and the bubbles were removed, the low shear force simulated microgravity (LSMMG) cultivation condition is generated by the HARVs' axis of rotation perpendicular to gravity direction, in contrast, the normal gravity (NG) cultivation condition is generated by the axis parallel to gravity direction[4]. There is a silicified gas-permeable membrane on the back of the HARVs, which can provide oxygen supply and prevent the leakage of liquid during bacterial culture.

Under the condition of $26^{\circ} \mathrm{C}$ and rotary speed of $25 \mathrm{rpm}, Y$. pestis was respectively grown to the middle exponential phase $\left(\mathrm{OD}_{620 \mathrm{~nm}}=1.0\right)$ for 24 hours under the SMG and NG conditions, then diluted to new HARV bioreactors for 40 consecutive passages of cultivation.

\section{Crystal violet staining}

The biofilm formation ability of $Y$. pestis is assessed by the crystal violet staining as follows. With the cultures being removed, the bioreactors were washed lightly with Phosphate Buffer Saline (PBS) and then were stained with $0.1 \%$ crystal violet solution for 20 min at indoor temperature.

In order to quantitatively analyze the difference of biofilm formation ability between SMG and NG group, the cultures were taken on a 24 -well plate at a dilution of $1: 100$ and incubated for 24 hours on a shaker at $37^{\circ} \mathrm{C}$ and $200 \mathrm{rpm}$. The optical density (OD) values were measured for each well at $620 \mathrm{~nm}$. Removed the bacterial solutions thoroughly and put the 24 -well plate at $80^{\circ} \mathrm{C}$ for $15 \mathrm{~min}$ to fix the biofilm. Each well was dyed with $0.1 \%$ crystal violet solution for $15 \mathrm{~min}$ and washed three times with PBS. Each well was added with $2 \mathrm{~mL}$ dimethyl sulfoxide solution to dissolve the crystal violet, and measured the OD values at $570 \mathrm{~nm}$. Finally, the ratios of $\mathrm{OD}_{570 \mathrm{~nm}}$ to $\mathrm{OD}_{620 \mathrm{~nm}}$ of each well were calculated.

\section{RAW 264.7 cells intracellular survival assay}


We intended to choose the RAW 264.7 cells evaluate the bacterial intracellular survival ability. The cultures grown to $\mathrm{OD}_{620 \mathrm{~nm}}=1.0$ under $\mathrm{SMG}$ and $\mathrm{NG}$ groups were added to 24-well plates that contains 70-80\% single-layer adherent RAW 264.7 cells at a MOI of $\sim 5$. At 0.5 hour past infection (hpi), gentamycin was added to kill the extracellular bacteria and the infected cells were lysed by Triton-X solution. Then living bacteria counting of the lysate was performed on the agar plates in triplicate. The above operations were repeated at 4, 6, $8 \mathrm{hpi}$.

\section{HeLa cells cytotoxicity/rounding assay}

HeLa cells were selected to evaluate the cytotoxicity of $Y$. pestis. Following the published methods for the HeLa cell rounding/cytotoxicity assay[12], saturated cultures of the two groups were added to infect the HeLa cells in six-well plates for 2.5 hours at a MOI of $\sim 50$. The HeLa cells cytotoxic effects were evaluated by observing the cell rounding phenotype through the microscopes.

However, considering that HeLa cells may be rounded due to crowded growth and errors existed in observing cell morphological changes with naked eyes, we intended to utilize the xCELLigence real-time cell analysis (RTCA, ACEA Biosciences Inc) system to analyze the dynamic and quantitative response profile of HeLa cells in vitro, and this technology is non-invasive and label-free. Cellular changes including cell number (cell index, $\mathrm{Cl}$ ) are recorded and analyzed via the RTCA system $[13,32,33]$. The principle of the RTCA system is to read the values of electronic impedance from the gold-plated sensor electrodes located on the bottom of microplates(E-plate). Electronic readings changes as the HeLa cells adhere or detach to the surface of the electrodes, cell index (CI) values are drawn via the software calculation[34].

HeLa cells were added into the 8-well microplates (E-plate) and cultured at $37^{\circ} \mathrm{C}$ with $5 \% \mathrm{CO}_{2}$ for 12 hours. The bacteria grown under SMG and NG conditions were added into the E-plate for 24 hours at a $\mathrm{MOI}$ of $\sim 5$. The cell index data was collected and analyzed by the RTCA Data Analysis Software 1.0 , and then the data was reanalyzed per 2 hours from 3-hour to 36-hour point using GraphPad Prism 5.0 software.

\section{BALB/c mice infection assay}

Saturated cultures grown under SMG and NG conditions were centrifuged at the rotary speed of 4500rpm, discarding the supernatant, and diluted to a concentration of one hundred microliters containing $100 \mathrm{CFU}$ or $10 \mathrm{CFU}$ with phosphate-buffered saline (PBS). The actual number of bacteria contained per 100 microliters is counted through dropping on the Hottinger's Agar plates. We randomly divided 32 8-week-old BALB/c female mice into 4 groups, and the diluted bacteria of 40 th passage were inoculated into each group with intraperitoneal route. The infected mice were monitored for 14 consecutive days and recorded the number of dead mice daily. The survival curves were drawn with GraphPad Prism 5.0 software, and the $p$-values less than 0.05 were considered statistically significant through log-rank (Mantel-Cox) test. The BABL/c mice used in this experiment were provided by Beijing Vital River Laboratory Animal Technology Co. Ltd [laboratory animal permit no. SCXK (Jing) 2016-0006]. All mice participating in this experiment were treated in accordance with the guidelines for the welfare and ethics of laboratory animals.

\section{RNA-seq-based transcriptional analysis}

The cultures in SMG and NG bioreactors were grown to saturation $\left(\mathrm{OD}_{620 \mathrm{~nm}}=1.0\right)$ as mentioned before. Each group was setting as three biological replicates. The mRNAs were obtained by removing the ribosomal RNA (rRNA) from the total RNA extracted by the PureLink ${ }^{\text {TM }}$ RNA Mini Kit (Invitrogen, Thermo Fisher Scientific, USA), and then used for creating cDNA library and deep sequencing. According to the values of FPKM (Fragments Per Kilobase Million), the ratio of transcript levels between SMG and NG groups were used as the logarithm to the base of 2 (Two-Fold Change), for example, a two-fold change value of 1.0 indicates 2 -fold greater expression of a certain gene. The differential values at least a 2-fold were applied to analyze the differential expression of genes according to the Y. pestis 91001 genome annotation.

\section{Quantitative real-time PCR}

The total RNA was extracted as described above, and purified by TURBO DNA-free ${ }^{\text {TM }}$ Kit (Invitrogen, Thermo Fisher Scientific, USA) upon the manufacturer's instructions. The total RNAs were reverse transcribed into cDNA as templates by SuperSript ${ }^{\text {TM }}$ Reverse Transcription Kit (Invitrogen, Thermo Fisher Scientific, USA). All primer pairs were designed to produce amplicons with expected sizes of 50-200 nt through the Primer Premier 5.0 software. Meanwhile, the highly expressed 16S ribosomal RNA gene was chose as the reference gene to draw the relative standard curve, in order to quantitatively compare and analyze differential expression levels of the target genes. The quantitative PCR (qPCR) was carried out in the Light Cycler R480 quantitative PCR instrument (Roche, CA, USA) and its software would calculate the relative fold change of target genes in the RNA samples of SMG and NG groups.

\section{Declarations}

Ethical approval and consent to participate

This study was carried out in compliance with the ARRIVE guidelines. The ethical approval of animals was obtained from the Beijing Institute of Microbiology and Epidemiology Ethics Committee. This article does not contain any studies with human participants performed by any of the authors

Consent for publication

Not applicable. 
Available of data and material

We guarantee that all data in the study are true and reliable. Data to support the findings in this study can be obtained from Ye Li.

Competing interests

All authors declare that they have no conflict of interests.

Funding

This study was funded by the Key Program of Logistics Research (Grant No. BWS18J012 \& BWS17J030).

Authors' contributions

YL performed experiments, analyzed the data and wrote the manuscript. LW $\mathbb{Y} \mathrm{C}$ and $\mathrm{YL}$ carried out the experiments and investigated the literature. RY provided overall directions throughout the study. YH designed the experiments, analyzed the data, provided overall directions and contributed to manuscript revisions.

Acknowledgements

Not applicable.

Author details

${ }^{1}$ State Key Laboratory of Pathogen and Biosecurity, Beijing Institute of Microbiology and Epidemiology, Beijing,100071, China

\section{References}

1. Buchheim JI, Matzel S, Rykova M, Vassilieva G, Ponomarev S, Nichiporuk I, Horl M, Moser D, Biere K, Feuerecker M et al: Stress Related Shift Toward Inflammaging in Cosmonauts After Long-Duration Space Flight. Front Physiol 2019, 10:85.

2. Sonnenfeld G, Shearer WJN: Immune function during space flight. 2002, 18(10):899-903.

3. Wilson JW, Ott CM, Honer zu Bentrup K, Ramamurthy R, Quick L, Porwollik S, Cheng P, McClelland M, Tsaprailis G, Radabaugh T et al: Space flight alters bacterial gene expression and virulence and reveals a role for global regulator Hfq. Proc Natl Acad Sci U S A 2007, 104(41):16299-16304.

4. Wang H, Yan Y, Rong D, Wang J, Wang H, Liu Z, Wang J, Yang R, Han Y: Increased biofilm formation ability in Klebsiella pneumoniae after short-term exposure to a simulated microgravity environment. MicrobiologyOpen 2016, 5(5):793-801.

5. Nickerson CA, Ott CM, Wilson JW, Ramamurthy R, Pierson DL: Microbial Responses to Microgravity and Other Low-Shear Environments. Microbiology and Molecular Biology Reviews 2004, 68(2):345-361.

6. Rosenzweig J, Abogunde O, Thomas K, Lawal A, Nguyen Y, Sodipe A, Jejelowo OJAm, biotechnology: Spaceflight and modeled microgravity effects on microbial growth and virulence. 2010, 85(4):885-891.

7. Nickerson CA, Ott CM, Mister SJ, Morrow BJ, Burns-Keliher L, Pierson DL: Microgravity as a novel environmental signal affecting Salmonella enterica serovar Typhimurium virulence. Infect Immun 2000, 68(6):3147-3152.

8. Wilson JW, Ramamurthy R, Porwollik S, McClelland M, Hammond T, Allen P, Ott CM, Pierson DL, Nickerson CA: Microarray analysis identifies Salmonella genes belonging to the low-shear modeled microgravity regulon. Proc Natl Acad Sci U S A 2002, 99(21):13807-13812.

9. Castro SL, Nelman-Gonzalez M, Nickerson CA, Ott CM: Induction of attachment-independent biofilm formation and repression of Hfq expression by lowfluid-shear culture of Staphylococcus aureus. App/ Environ Microbiol 2011, 77(18):6368-6378.

10. Singh NK, Wood JM, Karouia F, Venkateswaran K: Succession and persistence of microbial communities and antimicrobial resistance genes associated with International Space Station environmental surfaces. Microbiome 2018, 6(1):204.

11. Lawal A, Kirtley ML, van Lier CJ, Erova TE, Kozlova EV, Sha J, Chopra AK, Rosenzweig JA: The effects of modeled microgravity on growth kinetics, antibiotic susceptibility, cold growth, and the virulence potential of a Yersinia pestis ymoA-deficient mutant and its isogenic parental strain. Astrobiology 2013, 13(9):821-832.

12. Lawal A, Jejelowo OA, Rosenzweig JA: The effects of low-shear mechanical stress on Yersinia pestis virulence. Astrobiology 2010, 10(9):881-888.

13. Roshan Moniri M, Young A, Reinheimer K, Rayat J, Dai LJ, Warnock GL: Dynamic assessment of cell viability, proliferation and migration using real time cell analyzer system (RTCA). Cytotechnology 2015, 67(2):379-386.

14. Klaus DM, Howard HN: Antibiotic efficacy and microbial virulence during space flight. Trends Biotechnol 2006, 24(3):131-136.

15. Cornelis GR: The Yersinia Ysc-Yop 'type III' weaponry. Nat Rev Mol Cell Biol 2002, 3(10):742-752.

16. Torruellas J, Jackson MW, Pennock JW, Plano GV: The Yersinia pestis type III secretion needle plays a role in the regulation of Yop secretion. Mol Microbiol 2005, 57(6):1719-1733.

17. Rosqvist R, Forsberg A, Rimpilainen M, Bergman T, Wolf-Watz H: The cytotoxic protein YopE of Yersinia obstructs the primary host defence. Mol Microbiol 1990, 4(4):657-667.

18. Iriarte M, Cornelis GJMm: YopT, a new Yersinia Yop effector protein, affects the cytoskeleton of host cells. 1998, 29(3):915-929. 
19. Lindler LE, Klempner MS, Straley SC: Yersinia pestis pH 6 antigen: genetic, biochemical, and virulence characterization of a protein involved in the pathogenesis of bubonic plague. Infect Immun 1990, 58(8):2569-2577.

20. Sebbane F, Jarrett C, Gardner D, Long D, Hinnebusch BJPotNAoSotUSoA: Role of the Yersinia pestis plasminogen activator in the incidence of distinct septicemic and bubonic forms of flea-borne plague. 2006, 103(14):5526-5530.

21. Zimbler D, Schroeder J, Eddy J, Lathem WJNc: Early emergence of Yersinia pestis as a severe respiratory pathogen. $2015,6: 7487$.

22. Du Y, Rosqvist R, Forsberg A: Role of fraction 1 antigen of Yersinia pestis in inhibition of phagocytosis. Infect Immun 2002, 70(3):1453-1460.

23. Fetherston J, Kirillina O, Bobrov A, Paulley J, Perry RJI, immunity: The yersiniabactin transport system is critical for the pathogenesis of bubonic and pneumonic plague. 2010, 78(5):2045-2052.

24. Bearden SW, Fetherston JD, Perry RD: Genetic organization of the yersiniabactin biosynthetic region and construction of avirulent mutants in Yersinia pestis. Infect Immun 1997, 65(5):1659-1668.

25. Branda SS, Vik S, Friedman L, Kolter R: Biofilms: the matrix revisited. Trends Microbiol 2005, 13(1):20-26.

26. Costerton JW, Stewart PS, Greenberg EP: Bacterial biofilms: a common cause of persistent infections. Science 1999, 284(5418):1318-1322.

27. Mah T-FC, O'Toole GA: Mechanisms of biofilm resistance to antimicrobial agents. Trends in Microbiology 2001, 9(1):34-39.

28. Wilson JW, Ott CM, Ramamurthy R, Porwollik S, McClelland M, Pierson DL, Nickerson CA: Low-Shear modeled microgravity alters the Salmonella enterica serovar typhimurium stress response in an RpoS-independent manner. App/ Environ Microbio/ 2002, 68(11):5408-5416.

29. Hinnebusch BJ, Jarrett CO, Bland DM: "Fleaing" the Plague: Adaptations of Yersinia pestis to Its Insect Vector That Lead to Transmission. Annu Rev Microbio/ 2017, 71:215-232

30. Martínez L, Vadyvaloo VJFic, microbiology i: Mechanisms of post-transcriptional gene regulation in bacterial biofilms. $2014,4: 38$.

31. Song Y, Tong Z, Wang J, Wang L, Guo Z, Han Y, Zhang J, Pei D, Zhou D, Qin H et al: Complete genome sequence of Yersinia pestis strain 91001, an isolate avirulent to humans. DNA Res 2004, 11(3):179-197.

32. Cetin I, Topcul MR: Evaluation of the cytotoxic effect of Ly2109761 on HeLa cells using the xCELLigence RTCA system. Oncol Lett 2019, 17(1):683-687.

33. Pan T, Khare S, Ackah F, Huang B, Zhang W, Gabos S, Jin C, Stampfl M: In vitro cytotoxicity assessment based on $\mathrm{KC}(50)$ with real-time cell analyzer (RTCA) assay. Comput Biol Chem 2013, 47:113-120.

34. Qiao L, Xu Z, Zhao T, Zhao Z, Shi M, Zhao RC, Ye L, Zhang X: Suppression of tumorigenesis by human mesenchymal stem cells in a hepatoma model. Cell Res 2008, 18(4):500-507.

\section{Figures}





Figure 1

Biofilm measurement of Y. pestis grown under SMG or NG conditions by crystal violet staining. (A) The biofilms formed on the gas-permeable membrane on the back of bioreactors were stained by crystal violet. (B) With calculating the ratio of the OD 570nm to OD $620 \mathrm{~nm}$ values, the biofilm formation ability was quantitatively analyzed by GraphPad Prism 5.0 software ( $p=0.0067$, using Student $t$ test)).

(A)



(B)

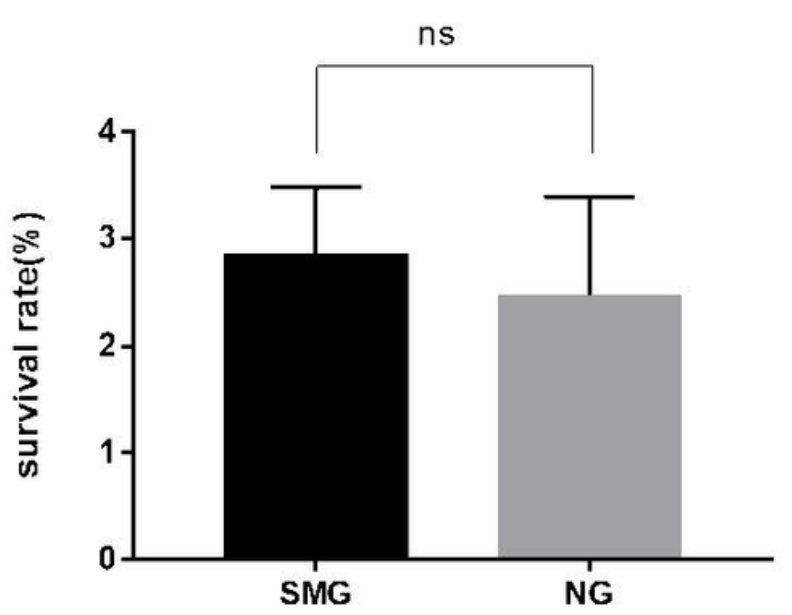

Figure 2

Survival dynamics of SMG- and NG-grown Y. pestis within RAW 264.7 macrophages. (A) RAW 264.7 cells were infected by cultures grown under SMG or NG condition at a $\mathrm{MOI}$ of $\sim 5$, and the viable bacteria in the cells at $0.5-, 4-, 6-, 8$-hour past infection (hpi) was respectively counted on the agar plates on which the 
cell lysates were plated. The survival percentage was expressed as the ratio of the amount of intracellular living bacteria at a certain-time point to the absolute amount of that at 30-min. (B) At 8-hpi, the differences in RAW 264.7 cells intracellular survival rate were not statistically significant.
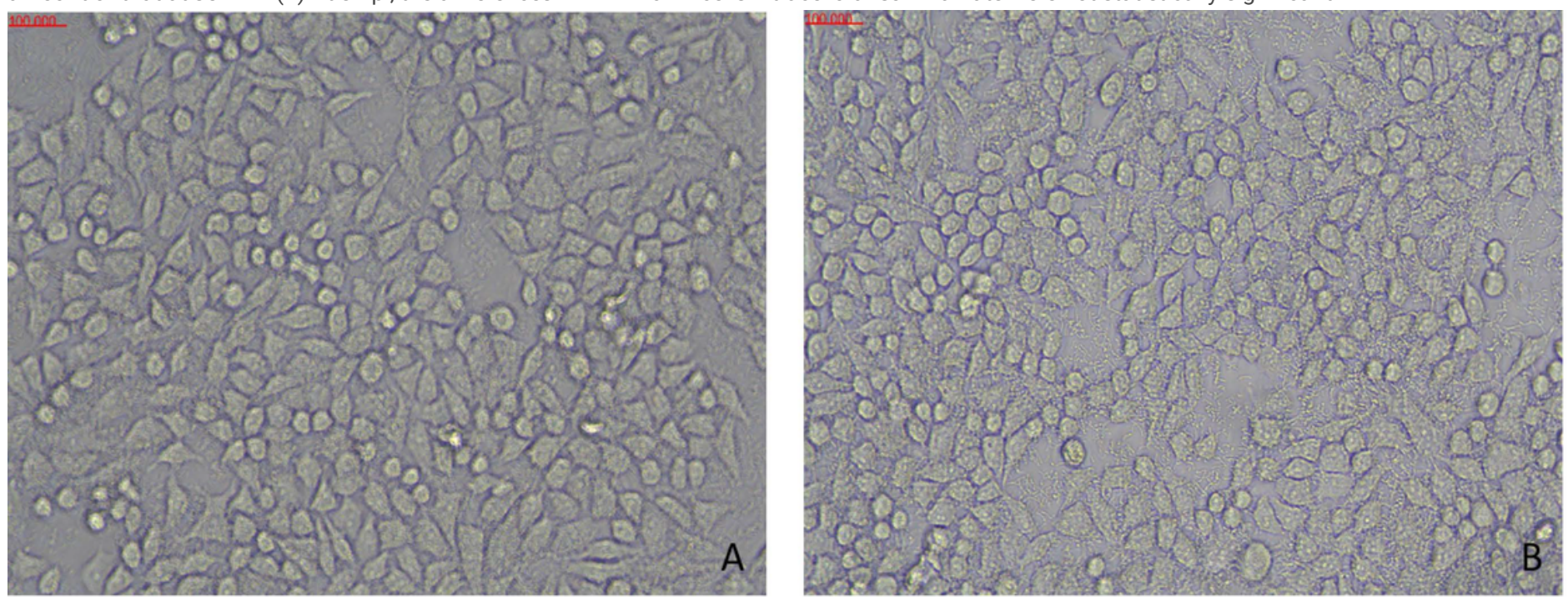

Figure 3

Monitoring of HeLa cells damages by comparing the proportion of rounding cells between SMG and NG group. The saturated HeLa cells were infected by Y. pestis grown under SMG (A) and NG (B) conditions at a MOI of 50. The photos were taken under optical microscope at 3-hour past infection (hpi). Based on the number of rounding cells in the vision field, rough ratios of rounding cells were calculated.

A
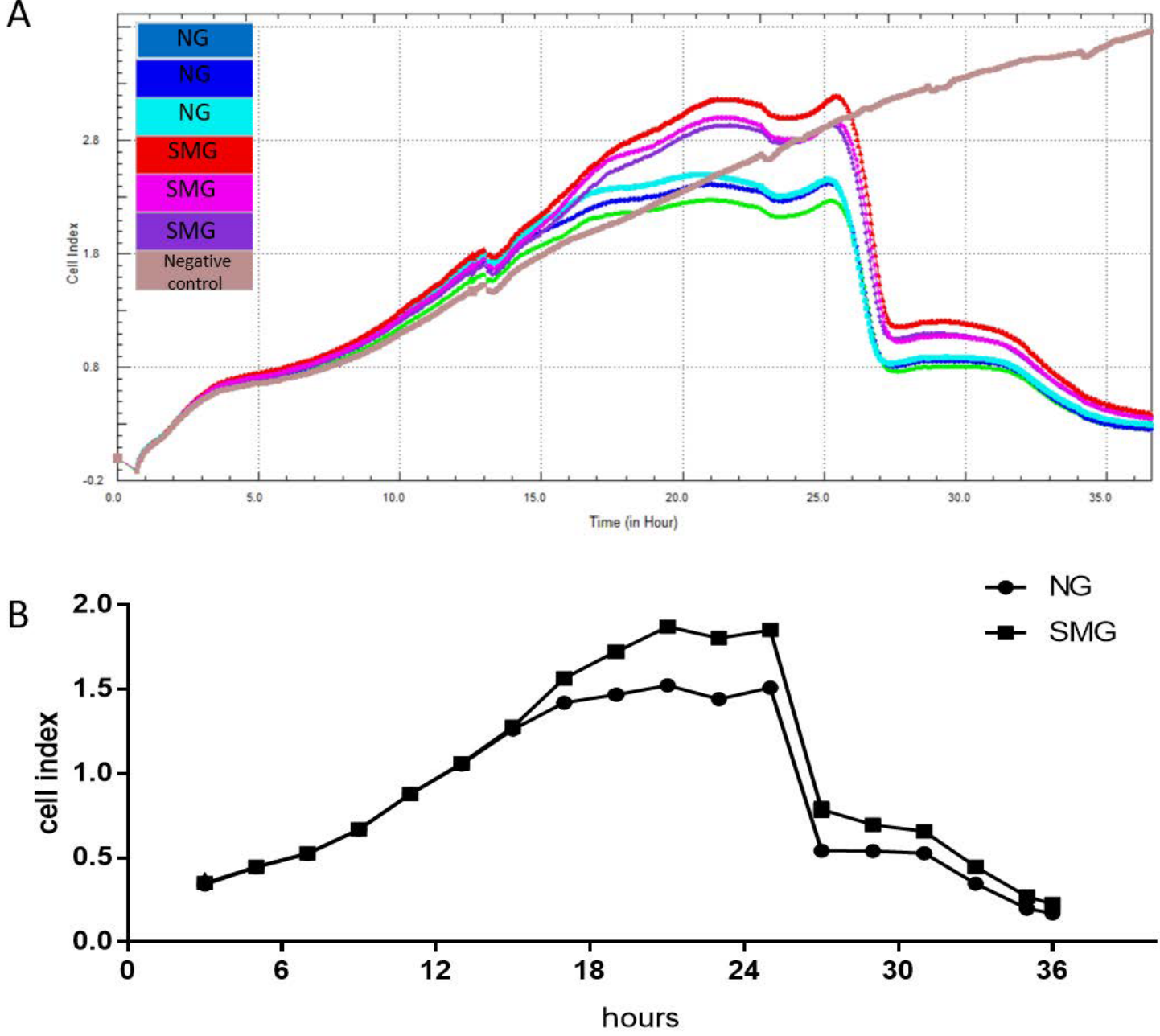
Quantitative Analysis of HeLa cells cytotoxicity by the Real-Time Cell Analysis (RTCA). HeLa cells were cultivated in the E-plate for $12 \mathrm{~h}$, and at a MOI of $\sim 5$, the cultures grown under SMG and NG conditions were respectively into each well in triplicate. (A) The last well containing HeLa cells only was regarded as the negative control. The raw data of cell index was collected by the RTCA Data Analysis Software 1.0. (B) After being normalized by index data at $12 \mathrm{~h}$, the cell index data was drawn per $2 \mathrm{~h}$ during the period of 3 to $36 \mathrm{~h}$ using GraphPad Prism 5.0 software.

A

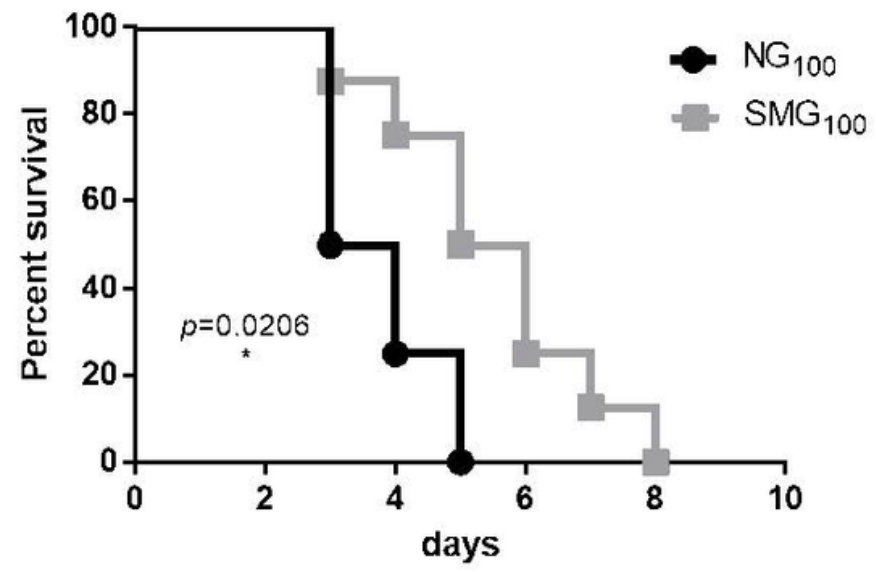

B

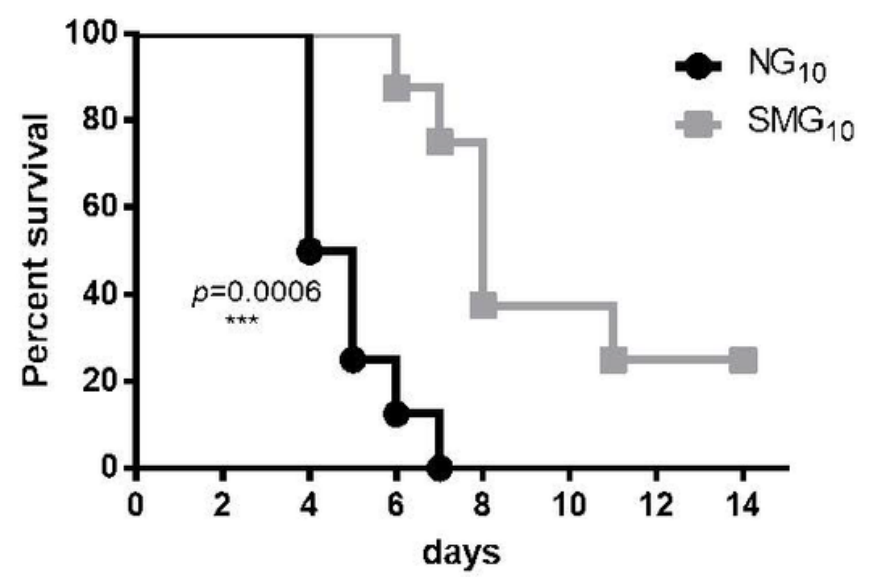

Figure 5

Survival curves of BABL/c mice infected by Y. pestis grown under SMG and NG conditions. (A) Each mouse was intraperitoneally inoculated by actual dose of 414 CFU of SMG group and 323 CFU of NG group. The survival curve had statistical significance $(p=0.206)$. (B) Mice were challenged with 42 CFU of SMG cultures and 36 CFU of NG cultures. The survival curve indicated a significant difference between two groups $(p=0.0006)$. Survival was monitored for 14 days.

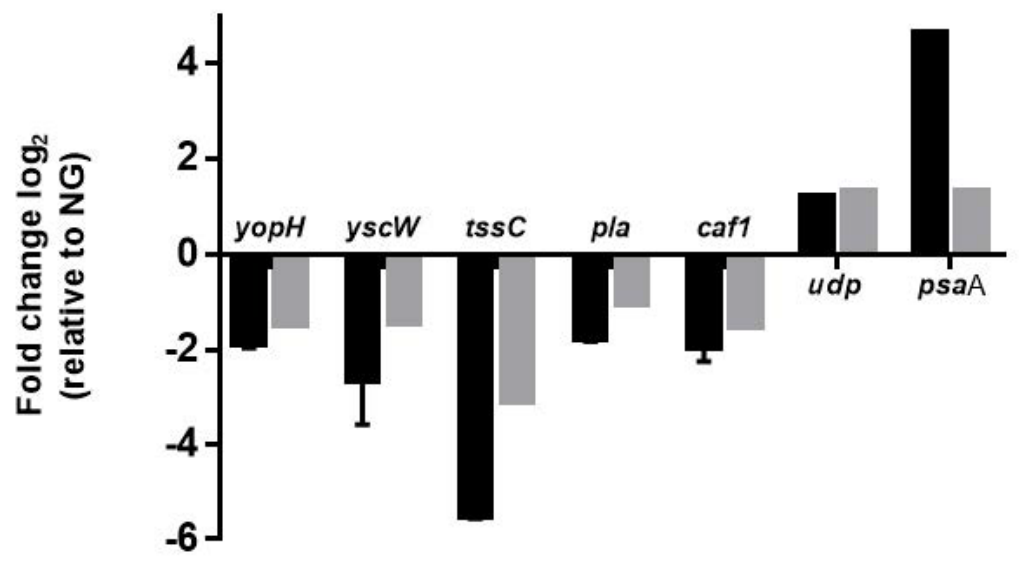

Figure 6

Relative expression of SMG group relative to NG group by qRT-PCR (black) and RNA-seq (gray). The RT-PCR result had a good consistency with the RNA-seq data. 